\title{
On Schwinger's formula for pair production
}

\author{
W. Dittrich \\ Institut für Theoretische Physik \\ Universität Tübingen \\ Auf der Morgenstelle 14 \\ D-72076 Tübingen \\ Germany \\ electronic address: qed.dittrich@uni-tuebingen.de
}

August 8, 2018

\begin{abstract}
We present some comments on Schwingers's calculation of electronpositron production in a prescribed constant electric field. The range of validity of $2 \operatorname{Im} \mathcal{L}^{(1)}(E)$ is discussed thoroughly and limiting cases are provided.
\end{abstract}

\section{Number of electron-positron pairs produced in a uniform electric field}

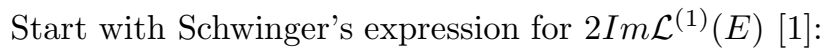

$$
\begin{aligned}
2 \operatorname{Im} \mathcal{L}^{(1)}=2 \frac{(e E)^{2}}{(2 \pi)^{3}} \underbrace{\sum_{n=1}^{\infty} \frac{1}{n^{2}} e^{-\frac{\pi n}{\beta}}}, \quad \beta=\frac{e E}{m^{2}} \\
=L i_{2}\left(e^{-\frac{\pi}{\beta}}\right), \text { Euler's dilogarithm }
\end{aligned}
$$

$\uparrow^{z} \boldsymbol{E}=$ const

$\hbar=c=1, \quad V=L^{3}$

In general for $\operatorname{spin} s=\frac{1}{2}$ and $s=0$ :

$$
\begin{aligned}
2 \operatorname{Im\mathcal {L}^{(1)}}(E) & =(2 s+1) \frac{(e E)^{2}}{(2 \pi)^{3}} \sum_{n=1}^{\infty} \frac{( \pm 1)^{n+1}}{n^{2}} e^{-n \frac{\pi}{\beta}} \\
& = \pm(2 s+1) \frac{(e E)^{2}}{(2 \pi)^{3}} L i_{2}\left( \pm e^{-\frac{\pi}{\beta}}\right) .
\end{aligned}
$$


Continuous phase space integration, how to count states:

$$
\int d^{3} \boldsymbol{p} \frac{V}{(2 \pi)^{3}} \cdot /=\int_{-\infty}^{+\infty} \frac{L}{2 \pi} d p_{1} \int_{-\infty}^{+\infty} \frac{L}{2 \pi} d p_{2} \int_{-\infty}^{+\infty} \frac{L}{2 \pi} d p_{3} . / .
$$

$t=\frac{p_{3}}{e E}$ in const. $E$-field: $d p_{3}=e E d t, \quad 0 \leq t \leq T$ or $\int_{-\infty}^{+\infty} d p_{3} \rightarrow e E T$.

Replace one factor $(e E)$ in (1.1) by $\frac{1}{T} \int_{-\infty}^{+\infty} d p_{3}$. Then

$$
\operatorname{Im}_{\mathcal{L}^{(1)}}(E) T=\frac{1}{(2 \pi)^{2}} \int_{-\infty}^{+\infty} d p_{3} \frac{(e E)}{(2 \pi)} \sum_{n=1}^{\infty} \frac{1}{n^{2}} e^{-\frac{\pi n}{\beta}}
$$

Rewrite the sum in (1.4):

$$
\begin{aligned}
& \frac{e E}{2 \pi} \sum_{n=1}^{\infty} \frac{1}{n^{2}} e^{-\frac{\pi}{\beta} n} \\
= & \sum_{n=1}^{\infty} \frac{1}{n} e^{-\frac{\pi}{\beta} n} \frac{1}{2} \frac{1}{\pi \frac{n}{e E}}, \quad \frac{1}{a}=\int_{0}^{\infty} d x e^{-a x}, \quad a=\pi \frac{n}{e E} \\
= & \sum_{n=1}^{\infty} \frac{1}{n} e^{-\frac{\pi}{\beta} n} \frac{1}{2} \int_{0}^{\infty} d\left(p_{\perp}^{2}\right) e^{-\pi \frac{n}{e E} p_{\perp}^{2}}, \quad d p_{\perp}^{2}=2 p_{\perp} d p_{\perp}, \quad \beta=\frac{e E}{m^{2}} \\
= & \sum_{n=1}^{\infty} \frac{1}{n} \int_{0}^{\infty} p_{\perp} d p_{\perp} \exp \left\{-\pi \frac{m^{2}+p_{\perp}^{2}}{e E} n\right\} \\
= & \int_{0}^{\infty} d p_{\perp} p_{\perp} \sum_{n=1}^{\infty} \frac{1}{n} \exp \left\{-\pi \frac{m^{2}+p_{\perp}^{2}}{e E} n\right\} \Longrightarrow
\end{aligned}
$$

Use $\ln (1-x)=-\sum_{n=1}^{\infty} \frac{1}{n} x^{n}, \quad|x|<1, \quad x=\exp \left\{-\pi \frac{m^{2}+p_{\perp}^{2}}{e E}\right\}$

$$
\begin{gathered}
\Longrightarrow=-\int_{0}^{\infty} d p_{\perp} p_{\perp} \ln \left(1-e^{-\pi \lambda_{p}}\right) \Longrightarrow, \quad \lambda_{p}=\frac{m^{2}+p_{\perp}^{2}}{e E} \\
e^{-\pi \lambda_{p}}=e^{-\pi \frac{m^{2}+p_{\perp}^{2}}{e E}}=\bar{n}_{p} .
\end{gathered}
$$

This important expression - also probability for tunneling - relates the imaginary part of the Lagrangian of the field to the mean number $\bar{n}_{p}$ of electron-positron 
pairs produced by the field in the state with given momentum and spin projection. $\bar{n}_{p}$ is degenerate with respect to spin (two) and momentum $p_{3}$ with $\frac{L_{3} \Delta p_{3}}{2 \pi \hbar}$ with $\Delta p_{3}=e E T$.

So we can continue to write (1.5):

$$
\Longrightarrow-\int_{0}^{\infty} d p_{\perp} p_{\perp} \ln \left(1-\bar{n}_{p}\right), \quad \int_{0}^{\infty} d p_{\perp} 2 \pi p_{\perp} \cdot / .=\int_{-\infty}^{\infty} d p_{1} d p_{2} \cdot / .
$$

Here then is the relation between $\operatorname{Im}^{(1)}(E)$ and $\bar{n}_{p}$ (insert $\hbar$ and $V=L^{3}$ ):

$$
\begin{aligned}
& \frac{2}{\hbar} \operatorname{Im} \mathcal{L}^{(1)} V T=-2 \int d^{3} \boldsymbol{p} \frac{V}{(2 \pi)^{3}} \ln \left(1-\bar{n}_{p}\right) \\
& \bar{n}_{p}=\exp \left\{-\pi \frac{m^{2}+p_{\perp}^{2}}{e E}\right\} .
\end{aligned}
$$

This is Nikishov's virial representation of the imaginary part of $\mathcal{L}^{(1)}(E)$ [2].

With the aid of (1.8) let us prove Nikishov's result for the mean number of pairs in four-volume $V T$ by counting states:

$$
\begin{aligned}
\bar{n} & =2 \int_{-\infty}^{+\infty} d p_{1} \frac{L}{(2 \pi)} \int_{-\infty}^{+\infty} d p_{2} \frac{L}{(2 \pi)} \int_{-\infty}^{e E L} d p_{3} \frac{T}{(2 \pi)} \bar{n}_{p} \Longrightarrow \\
& =2 \frac{L^{2}}{(2 \pi)^{2}} 2 \pi \int_{0}^{\infty} p_{\perp} d p_{\perp} \frac{T}{2 \pi} e E L e^{-\pi \lambda_{p}} \\
& =\frac{(e E)}{(2 \pi)^{2}} V T \int_{0}^{\infty} d p_{\perp}^{2} e^{-\pi \frac{p_{1}^{2}}{e E}} e^{-\frac{\pi}{\beta}}=\frac{e E}{(2 \pi)^{2}} V T \frac{1}{\pi \frac{1}{e E}} e^{-\frac{\pi}{\beta}} .
\end{aligned}
$$

Therefore

$$
\bar{n}=2 \frac{(e E)^{2}}{(2 \pi)^{3}} V T e^{-\frac{\pi}{\beta}}, \quad \beta=\frac{e E}{m^{2}},
$$

which is Nikishov's result for the mean number of pairs produced in volume $V=L^{3}$ during time $T$. For Bose particles the factor 2 is suppressed.

Introducing

$$
\xi=\exp \left\{-\frac{\pi m^{2}}{e E}\right\}, \quad \gamma=V T \frac{(e E)^{2}}{4 \pi^{3}}
$$

we also can write

$$
\bar{n}=\gamma \xi
$$


Formula (1.9) is an approximation of the following expression for $n=1$ :

$$
\int d^{3} p \frac{V}{(2 \pi)^{3}} \sum_{n=1}^{\infty} \bar{n}_{p}^{n}
$$

Furthermore the well-known vacuum persistence probability $\left|\left\langle O_{+} \mid O_{-}\right\rangle\right|^{2}$ is given by

$$
\begin{aligned}
\left|\left\langle O_{+} \mid O_{-}\right\rangle\right|^{2} & =p_{0}=\prod_{s, p}\left(1-e^{-\pi \lambda_{p}}\right) \\
& =\prod_{s, p}\left(1-e^{-\pi \frac{m^{2}+p_{\perp}^{2}}{e E}}\right)=e^{-2 I m \mathcal{L}^{(1)}(E) V T} \\
& =\exp \left\{-V T \frac{(e E)^{2}}{4 \pi^{3}} \sum_{n=1}^{\infty} \frac{1}{n^{2}} e^{-n \frac{\pi m^{2}}{e E}}\right\} \\
& =\exp \left\{-\gamma L i_{2}(\xi)\right\} .
\end{aligned}
$$

Schwinger writes in his brilliant article [1] as well as in his 2nd volume on "sources, particles and fields": "We recognize in $2 \operatorname{Im} \mathcal{L}^{(1)}$ a measure in the probability, per unit time and unit spatial volume, that an electron-positron pair has been created." This statement is only true for very weak fields $\left(e E \ll m^{2}\right)$ in which case the contributions of the $n=2,3, \ldots$ terms in the sum of (1.1) can be neglected:

$$
2 \operatorname{Im}^{(1)}\left(e E \ll m^{2}\right)=2 \frac{(e E)^{2}}{(2 \pi)^{3}} e^{-\frac{\pi}{\beta}} \equiv \frac{\bar{n}}{V T}=\frac{\gamma \xi}{V T} \simeq \frac{\gamma L}{V T} ; \quad L=-\ln (1-\xi),
$$

which is identical to Nikishov's result. Here, in order to save at least part of Schwinger's statement, we are being a bit casual since $\bar{n}$ is not a probability but an average number. To be more specific let us start with (1.12):

$$
\begin{aligned}
& 2 \int d^{3} \boldsymbol{p} \frac{V}{(2 \pi)^{3}} \sum_{n=1}^{\infty}\left(\bar{n}_{p}\right)^{n}, \quad \int d p_{3}=T e E \\
= & \left.V \frac{T e E}{4 \pi^{3}} \int_{-\infty}^{+\infty} d p_{1} \int_{-\infty}^{+\infty} d p_{2} \sum_{n=1}^{\infty}\left(\bar{n}_{p}\right)\right)^{n}, \quad \int d p_{1} \int d p_{2}=2 \pi \int_{0}^{\infty} d p_{\perp} p_{\perp} \\
= & V T \frac{(e E)}{2 \pi^{2}} \int_{0}^{\infty} p_{\perp} d p_{\perp} \sum_{n=1}^{\infty} e^{-\pi \lambda_{p} n}, \bar{n}_{p}=e^{-\pi \lambda_{p}}, \lambda_{p}=\frac{m^{2}+p_{\perp}^{2}}{e E}, \beta=\frac{e E}{m^{2}} \\
= & V T \frac{(e E)}{2 \pi^{2}} \sum_{n=1}^{\infty} e^{-\frac{\pi}{\beta} n} \frac{1}{2} \int_{0}^{\infty} d p_{\perp}^{2} e^{-\pi \frac{n}{e E} p_{\perp}^{2}} \\
= & V T \frac{(e E)}{2 \pi^{2}} \sum_{n=1}^{\infty} e^{-\frac{\pi}{\beta} n} \frac{1}{2 \frac{\pi n}{e E}}=V T \frac{(e E)^{2}}{4 \pi^{3}} \sum_{n=1}^{\infty} \frac{1}{n} e^{-\frac{\pi m^{2}}{e E} n} \\
= & V T \frac{(e E)^{2}}{4 \pi^{3}}\left[-\ln \left(1-e^{-\pi \frac{m^{2}}{e E}}\right)\right]=: \frac{p_{1}}{p_{0}}=\gamma L .
\end{aligned}
$$


Finally

$$
\begin{aligned}
& p_{1}=V T \frac{(e E)^{2}}{4 \pi^{3}}\left[-\ln \left(1-e^{-\pi \frac{m^{2}}{e E}}\right)\right] \cdot \exp \left\{-V T \frac{(e E)^{2}}{4 \pi^{3}} \sum_{n=1}^{\infty} \frac{1}{n^{2}} e^{-n \frac{\pi m^{2}}{e E}}\right\} \\
& p_{1}=\gamma L p_{0}, \quad L=-\ln (1-\xi), \quad p_{0}=\exp \left\{-\gamma L i_{2}(\xi)\right\} .
\end{aligned}
$$

So far we have

$$
\begin{aligned}
p_{0} & =\exp \left\{-\gamma L i_{2}(\xi)\right\}, \quad \text { Schwinger's vaccuum persistence probability } \\
p_{1} & =\gamma L p_{0} \\
\bar{n} & =\gamma \xi
\end{aligned}
$$

Let's denote by $\alpha=(\boldsymbol{p}, s)$ the quantum numbers of the electron states. Then we can write our vacuum-to-vacuum probability as

$$
p_{0}=\prod_{\alpha}\left(1-\bar{n}_{\alpha}\right)
$$

In an electric field any number of pairs can be produced, so the probability that there are $n=0,1,2, \ldots$ electron-positron pairs shows up in the series

$$
\begin{aligned}
\sum_{n=0}^{\infty} p_{n}= & \prod_{\alpha}\left(1-\bar{n}_{\alpha}\right)+\sum_{\alpha} \bar{n}_{\alpha} \prod_{\beta \neq \alpha}\left(1-\bar{n}_{\beta}\right) \\
& +\frac{1}{2 !} \sum_{\alpha \neq \beta} \bar{n}_{\alpha} \bar{n}_{\beta} \prod_{\gamma \neq \alpha, \beta}\left(1-\bar{n}_{\gamma}\right)+\ldots=1 .
\end{aligned}
$$

In our case, each of the quantities $p_{n}$ describes the propability for the number $n=0,1,2, \ldots$ electron-positron pairs in four-volume. The first terms were calculated above:

$$
\begin{aligned}
& p_{0}=\exp \left\{-\gamma L i_{2}(\xi)\right\} \\
& p_{1}=\gamma L p_{0} .
\end{aligned}
$$

The next and followers for the numbers $n$ were calculated by Krivoruchenko:

$$
p_{2}=\frac{\gamma}{2}\left(\gamma L^{2}+L-\frac{\xi}{1-\xi}\right) p_{0} \quad \text { etc. }
$$

It is highly interesting to follow Krivoruchenko's paper [3] and find out that in electric fields of supercritical strength $|e E|>\frac{\pi m^{2}}{\ln 2}$, the unitary condition (1.16), $\sum_{n=0}^{\infty} p_{n}=1$, changes into an asymptotic divergence, i.a. the positive definiteness of the probability is violated. This divergence indicates a failure of the continuum limit approximation, i.e. by the replacement of the discrete sum by the integral over the phase space:

$$
\sum_{\alpha} . / . \rightarrow 2 \int \frac{V}{(2 \pi)^{3}} d^{3} \boldsymbol{p} \ldots=V T|e E| \int \frac{2 d^{2} p_{\perp}}{(2 \pi)^{3}} \ldots
$$




\section{Schwinger's formula for $\operatorname{Im}^{(1)}(E)$ the long way, i.e., without using the residue theorem.}

Take the formula (5.27) or equivalently (6.33) of the "Lecture Notes 220" on "Effective Lagrangians in QED" by Dittrich and Reuter [4, 5]:

$$
\begin{aligned}
\mathcal{L}^{(1)}(B)= & -\frac{1}{32 \pi^{2}}\left\{\left(2 m^{4}-4 m^{2}(e B)+\frac{4}{3}(e B)^{2}\right)\left[1+\ln \left(\frac{m^{2}}{2 e B}\right)\right]\right. \\
& \left.+4 m^{2}(e B)-3 m^{4}-(4 e B)^{2} \zeta^{\prime}\left(-1, \frac{m}{2 e B}\right)\right\} .
\end{aligned}
$$

This can also be written in the form

$$
\begin{aligned}
& \mathcal{L}^{(1)}(B) \\
= & -\frac{1}{32 \pi^{2}}\left\{-3 m^{4}+4(e B)^{2}\left(\frac{1}{3}-4 \zeta^{\prime}(-1)\right)+4 m^{2}(e B) \ln 2 \pi-1\right) \\
& -2 m^{4} \ln \frac{2 e B}{m^{2}}-4 m^{2}(e B) \ln \frac{2 e B}{m^{2}}-\frac{4}{3}(e B)^{2} \ln \frac{2 e B}{m^{2}} \\
& \left.-16(e B)^{2} \int_{1}^{1+\frac{m^{2}}{2 e B}} d x \ln \Gamma(x)\right\} .
\end{aligned}
$$

Introducing the critical field strength $B_{e r}=\frac{m^{2}}{e}$ and measuring the magnetic field in this unit, we can rewrite the last expression as

$$
\begin{aligned}
\mathcal{L}^{(1)}(B)= & \frac{\alpha}{2 \pi}\left\{\frac{3}{4}-B(\ln (2 \pi)-1)-B^{2}\left(\frac{1}{3}-4 \zeta^{\prime}(-1)\right)\right. \\
& \left.+\left(\frac{1}{2}+B+\frac{1}{3} B^{2}\right) \ln (2 B)+4 B^{2} \int_{1}^{1+\frac{1}{2 B}} \ln (\Gamma(x)) d x\right\}
\end{aligned}
$$

For a pure electric field the Lagrangian is likewise given by

$$
\begin{aligned}
\mathcal{L}^{(1)}(E)= & -\frac{1}{32 \pi^{2}}\left\{\left(2 m^{4}+4 i m^{2} e E-\frac{4}{3} e^{2} E^{2}\right)\left(\ln \left(i \frac{m^{2}}{2 e E}\right)+1\right)\right. \\
& \left.-3 m^{4}-4 i m^{2} e E+16 e^{2} E^{2} \zeta^{\prime}\left(-1, i \frac{m^{2}}{2 e E}\right)\right\} .
\end{aligned}
$$


It takes a litte practice to separate this formula into its real and imaginary part:

$$
\begin{aligned}
\mathcal{L}^{(1)}(E)= & \frac{\alpha}{2 \pi}\left\{\frac{3}{4}+\frac{1}{2} \ln (2 E)-\frac{\pi}{2} E+E^{2}\left(\frac{1}{3}-4 \zeta^{\prime}(-1)\right)-\frac{1}{3} E^{2} \ln (2 E)\right. \\
& \left.+4 E^{2} \int_{0}^{1 / 2 E} \operatorname{Im} \ln (\Gamma(1+y)) d y\right\} \\
& +i \frac{\alpha}{2 \pi}\left\{-\frac{\pi}{4}-E \ln (2 E)+E(\ln (2 \pi)-1)\right. \\
& \left.+\frac{\pi}{6} E^{2}-4 E^{2} \int_{0}^{1 / 2 E} \operatorname{Re} \ln (\Gamma(1+i y)) d y\right\} .
\end{aligned}
$$

With the aid of the relation (Gradshteyn / Ryzhik)

$$
\begin{aligned}
\operatorname{Re} \ln (\Gamma(1+i y)) & =\ln |\Gamma(1+i y)|=\frac{1}{2} \ln [\Gamma(1+i y) \Gamma(1-i y)] \\
& =-\frac{1}{2} \ln \frac{\sinh (\pi y)}{\pi y}
\end{aligned}
$$

and an integration by parts

$$
\int_{0}^{1 / 2 E} 1 \cdot \ln \frac{\sinh (\pi y)}{\pi y} d y=\frac{1}{2 E} \ln \left[\frac{2 E}{\pi} \sinh \frac{\pi}{2 E}\right]-\int_{0}^{1 / 2 E}[\pi y \operatorname{coth}(\pi y)-1] d y,
$$

we obtain

$$
\begin{aligned}
\operatorname{Im} \mathcal{L}^{(1)}(E)= & \frac{\alpha}{2 \pi}\left\{-\frac{\pi}{4}+E \ln 2+\frac{\pi}{6} E^{2}+E \ln \left(\sinh \frac{\pi}{2 E}\right)\right. \\
& \left.-2 E^{2} \int_{0}^{1 / 2 E} \pi y \operatorname{coth}(\pi y) d y\right\} .
\end{aligned}
$$

Let's change the variable $x=\pi y$ and evaluate the integral on the right-hand side with the use of [Wolfram Mathematica online integrator]

$$
\begin{aligned}
\int x \operatorname{coth}(x) d x & =\frac{1}{2}\left(x\left(x+2 \ln \left(1-e^{-2 x}\right)\right)-L i_{2}\left(e^{-2 x}\right)\right) \\
& =\frac{1}{2}\left(x\left(x+2 \ln \left(2 e^{-x} \sinh (x)\right)-L i_{2}\left(e^{-2 x}\right)\right) .\right.
\end{aligned}
$$

With integration limits we arrive at

$$
\begin{aligned}
\frac{1}{\pi} \int_{0}^{\frac{\pi}{2} E} x \operatorname{coth}(x) d x= & \frac{1}{2 \pi}\left[\frac{\pi}{2 E}\left(\frac{\pi}{2 E}+2 \ln 2-\frac{\pi}{E}+2 \ln \left(\sinh \left(\frac{\pi}{2 E}\right)\right)\right)\right. \\
& \left.-L i_{2}\left(e^{-\frac{\pi}{E}}\right)+\frac{\pi^{2}}{6}\right] .
\end{aligned}
$$


We substitute this in our last expression for $\operatorname{Im}^{(1)}(E)$ and obtain

$$
\begin{aligned}
\operatorname{ImL}^{(1)}(E)= & \frac{\alpha}{2 \pi}\left\{-\frac{\pi}{4}+E \ln 2+\frac{\pi}{6} E^{2}+E \ln \left(\sinh \left(\frac{\pi}{2 E}\right)\right)\right. \\
& \left.+\frac{\pi}{4}-E \ln 2-E \ln \left(\sinh \left(\frac{\pi}{2 E}\right)\right)+\frac{E^{2}}{\pi} L i_{2}\left(e^{-\frac{\pi}{E}}\right)-\frac{\pi}{6} E^{2}\right\} \\
= & \frac{\alpha}{2 \pi} \frac{E^{2}}{\pi} L i_{2}\left(e^{-\frac{\pi}{E}}\right) \\
\left(L i_{2}\left(e^{-\frac{\pi}{E}}\right)=\right. & \sum_{n=1}^{\infty} \frac{1}{n^{2}} e^{-\frac{\pi}{E} n} ; \\
& \text { in units of } \left.\quad E_{c r}=\frac{m^{2}}{e}: \sum_{n=1}^{\infty} \frac{1}{n^{2}} e^{-\frac{m^{2} \pi}{e E} n}\right) .
\end{aligned}
$$

Finally we obtain J.S.'s famous result $\left(\alpha=\frac{e^{2}}{4 \pi}\right)$ :

$$
\operatorname{Im}^{(1)}(E)=\frac{\alpha}{2 \pi^{2}} E^{2} \sum_{n=1}^{\infty} \frac{1}{n^{2}} e^{-\frac{m^{2} \pi}{e E} n} .
$$

At last we might add the result for the real part [6]:

$$
\begin{aligned}
R e \mathcal{L}^{(1)}(E)= & -\frac{e^{2} E^{2}}{4 \pi^{4}}\left(C+\ln \frac{\pi m^{2}}{e E}\right) \sum_{n=1}^{\infty} \frac{1}{n^{2}} \cosh \left\{n \frac{\pi m^{2}}{e E}\right\} \\
& -\frac{e^{2} E^{2}}{4 \pi^{4}} \sum_{n=1}^{\infty} \frac{\ln n}{n^{2}} \cosh \left\{n \frac{\pi m^{2}}{e E}\right\},
\end{aligned}
$$

where $C$ is the Euler-Mascheroni constant.

\section{Acknowledgement}

The author wishes to thank M. I. Krivoruchenko for critical remarks and several interesting discussions.

\section{References}

[1] J. Schwinger, Phys. Rev. 82, 664 (1951).

Particles, Sources, and Fields, Volume II, p. 134, Addison-Wesley Publishing Company 1989

[2] Sov. Phys. JETP 30, 660 (1970)

[3] M. I. Krivoruchenko, Phys. Rev. D 86, 027704 (2012) [arXiv:1206.3836 [hep-ph]]. 
[4] W. Dittrich, J. Phys. A Vol. 9, No. 7, 1171 (1976)

Replace in this reference in (3.25) $\zeta^{\prime}$ by $-\frac{1}{2} \zeta^{\prime}$ and $\ln \frac{m^{2}}{e H}$ by $\ln \frac{m^{2}}{2 e H}$.

[5] W. Dittrich and M. Reuter, Effective Lagrangians in Quantum Electrodynamics (Springer, New York, 1985)

[6] R. Soldati, J. Phys. A 44, 305401 (2011) [arXiv:1104.0468 [hep-th]]. 